\section{ON THE POLYMORPH-SENSITIVE CHEMICAL REACTIVITY OF} DIAZINES

A. Katrusiak ${ }^{1}$ Anna Katrusiak ${ }^{2}$

${ }^{1}$ Adam Mickiewicz University Faculty of Chemistry Grunwaldzka 6 POZNAN 60-780 POLAND ${ }^{2}$ Faculty of Pharmacy, Karol Marcinkowski Medical Academy, Grunwaldzka 6, 60-780 Poznan, Poland

Differences between polymorphs are usually associated with their physical properties, like melting point, solubility, absorption (colour), electric-current conductivity, ferroelectricity, etc. Recently we have also observed different chemical reactivity of two biologically important diazines. Two polymorphs of the first compound differ in ionicity: either molecules or anions and cations are present in the crystalline phases. It has been shown that it is in the ionic state that the dimerization takes place. The polymorphs of the second diazine were found to form different products of the reaction of chlorination. The structural origins of the different chemical reactivity of the polymorphs will be presented and their consequences discussed. A new understanding bearing on the chemical transformations of like substances emerge from this study. It is apparent that the transformations of these molecules are even more likely in the living tissue, but it was owing to polymorphism that they have been detected

\section{Keywords: POLYMORPHISM, REACTIVITY, DIAZINES}

\section{Acta Cryst. (2002). A58 (Supplement), C324 \\ OBSERVATION OF PHOTO-EXCITED STRUCTURE OF A} PLATINUM COMPLEX ANION

N. Yasuda M. Kanazawa H. Uekusa Y. Ohashi

Tokyo Institute of Technology Department of Chemistry and Materials Science 2-12-1, Ookayama, Meguro-Ku TOKYO 152-8551 JAPAN

It was reported that the crystal lattice of the platinum complex, $\left[\mathrm{Bu}_{4} \mathrm{~N}\right]\left[\mathrm{Pt}_{2}\right.$ (pop)] (pop $=\left[\mathrm{P}_{2} \mathrm{O}_{5} \mathrm{H}_{2}\right]^{2-}$ ), contracted on exposure to visible light and return to the original cell dimensions when the irradiation was stopped. The contraction was assumed to be due to the formation of the excited state by photo irradiation. In order to examine the photo-excited structure of the platinum complex anion, the structures of several related platinum complex crystals during the irradiation were analyzed by X-rays at low temperatures. The crystals have tetrabutylammonium, tetrapentylammonium or benzyl(triethyl)ammonium as cations. In some crystals solvent molecules are also included. The preliminary structures analyzed by $\mathrm{X}$-rays revealed that the chemical formula of the platinum complex is not $\left[\mathrm{Pt}_{2}\right.$ (pop)] but $\left[\mathrm{Pt}_{2} \mathrm{H}_{2}\right.$ (pop)]. This means that two protons are attached to the phosphate groups, which are clearly assigned by the P-O distances. Each crystal was irradiated with the xenon lamp at low temperatures. The unit-cell volume decreased significantly. The three-dimensional intensity data were collected with the light on and off. The structures were analyzed and refined. The significant change in molecular structures are observed between the light on and off; the Pt-Pt distance decreased significantly when the light was on. The other distances were insignificantly changed. From the theoretical calculation, the Pt-Pt distance should decrease if the Pt atom was excited. These results suggest that the Pt atom at the excited state can be observed by usual X-ray analysis.

\section{Keywords: PHOTO-EXCITED STRUCTURE X-RAY ANALYSIS PLATINUM COMPLEX}

Acta Cryst. (2002). A58 (Supplement), C324

\section{CRYSTAL STRUCTURES AND PHOTOCONDUCTIVITY OF CHARGE GENERATION MATERIALS}

Y. Kojima C. Sasaki T. Shoda T. Ohashi

Mitsubishi Chemical Corporation CACs Yokohama Laboratory 1000,

Kamoshida-Cho, Aoba-Ku YOKOHAMA 227-8502 JAPAN

Oxotitanium Phthalocyanine [TiOPc] has been developed as a charge generation material (CGM) of electrophotographic photoreceptors. TiOPc has four polymorphs (form I, II, C and Y) whose photosensitivity depends on the crystal structures (photosensitivity: form Y > I > II > C). Only the crystal structures of more stable polymorphs (form I and II) have been reported. We succeeded to obtain TiOPc form $\mathrm{C}$ crystals by solid-solvent growth method and determined the crystal structure. The stacking of phthalocyanine planes along $\mathrm{c}$-axis is formed in form $\mathrm{C}$. The crystal structure of form $\mathrm{C}$ is obviously different from those of form I and II. We also determined cell parameters and space group of form $\mathrm{Y}$ from $\mathrm{X}$-ray powder diffraction data. The results suggest that the packing of form $\mathrm{Y}$ is similar to that of form I. Dihydroxysilicon phthalocyanine $\left[\mathrm{Si}(\mathrm{OH})_{2} \mathrm{Pc}\right]$ was synthesized for new CGMs. We found out $\mathrm{Si}(\mathrm{OH})_{2} \mathrm{Pc}$ has three polymorphs (Phase I, II and III). Phase II and III show suitable photosensitivity for laser printers, while Phase I has no sensitivity. The three polymorphs of $\mathrm{Si}(\mathrm{OH})_{2} \mathrm{Pc}$ have never grown in large enough crystals for single crystal analysis and we carried out ab-initio X-ray powder structure analysis using synchrotron radiation. The main difference in packings was revealed between the photosensitive and insensitive crystals, Phase II and III crystals have the herringbone structure, on the other hand in Phase I all molecules form the parallel stack columns. We discuss the relationship between the intermolecular interactions and the photosensitivity of TiOPc and $\mathrm{Si}(\mathrm{OH})_{2} \mathrm{Pc}$.

\section{Keywords: POLYMORPH PHOTOSENSITIVITY AB-INITIO POWDER STRUCTURE ANALYSIS}

\section{Acta Cryst. (2002). A58 (Supplement), C324 \\ LOCAL STRUCTURE AND DYNAMICS OF PROTONS IN LETOVICITE: AN NMR SPECTROSCOPIC STUDY A. Engelhardt $^{1}$ M. Fechtelkord ${ }^{1}$ J.-C. Buhl ${ }^{1}$ L. Schwalowsky ${ }^{2}$ U. Bismayer ${ }^{2}$ ${ }^{1}$ Universitaet Hannover Institut Fuer Mineralogie Welfengarten 1 HANNOVER 30167 GERMANY ${ }^{2}$ Mineralogisch-Petrographisches Institut, Universitaet Hamburg, Grindelallee 48, D-20146 Hamburg, Germany}

Letovicite belongs to the well-studied class of improper ferroelastic materials of general formula $\mathrm{M}_{3} \mathrm{H}\left(\mathrm{XO}_{4}\right)_{2}$ with $\mathrm{M}=\mathrm{K}, \mathrm{Rb}, \mathrm{Cs}, \mathrm{NH}_{4}$ and $\mathrm{X}=\mathrm{S}$, Se. The monoclinic room temperature phase with space group $C 2 / c$ undergoes a phase transition to the trigonal paraelastic high temperature phase at $\mathrm{TC}=413 \mathrm{~K}$. The formation of domain boundaries first occurs at a temperature of $20 \mathrm{~K}$ below TC. In addition, superionic proton conductivity is observed in the paraelastic phase. Solid State NMR spectroscopy is an ideal method to investigate the dynamics and local structure of the protons. ${ }^{1} \mathrm{H}$ MAS NMR spectroscopy is capable to separate the signal contributions of the free acidic and the ammonium protons due to their different chemical shift [1]. The alteration of observed signal linewidths depicts the change of the proton dynamics at different temperatures. While the motional changes of the acidic protons accompany the phase transition the onset of motion of the ammonium tetrahedra occurs $10-15 \mathrm{~K}$ below the phase transition temperature. ${ }^{2} \mathrm{D}{ }^{2} \mathrm{H}$ NMR powder spectra exhibit characteristic ridge patterns originating from the singularities of the ${ }^{2} \mathrm{D}$ lineshape for discrete jump motions and reorientation angles, thus yielding information about the type of motion. The information of two-dimensional spectra is used to simulate one-dimensional static ${ }^{2} \mathrm{H} N \mathrm{NMR}$ spectra. The form of the static lineshape at different temperatures depends on the type of motion and the jump rate and thus gives information about the mechanism of the phase transition and the contribution to the superionic conductivity.

[1] M.Fechtelkord, A. Engelhardt, J.-C. Buhl, L. Schwalowsky, U. Bismayer. Solid State NMR, 17, (2000) 76-88.

\section{Keywords: NMR SPECTROSCOPY PHASE TRANSITION PROTON} DYNAMICS 